\title{
Prospect for VLBI Network Extension: the First Results of an Ad-hoc S2 Array Experiments
}

Igor Molotov, Sergey Likhachev, Andrey Chuprikov, Boris Lipatov, Alexander Dementiev

Astro Space Center, Lebedev Physical Institute, Moscow, Russia

Wayne Cannon

Space Geodynamics Laboratory, North York, Canada

Sean Dougherty, David Del Rizzo

Dominion Radio Astrophysical Observatory, Penticton, Canada

Carlo Stanghellini, Corrado Trigilio

Instituto di Radioastronomia, Bologna, Italy

Xiaoyu Hong, Xinyong Huang, Liang Shiguang

Shanghai Astronomical Observatory, Shanghai, China

Jonathan Quick, George Nicolson

Hartebeesthoek Radio Astronomy Observatory, Krugersdorp, South Africa

\begin{abstract}
The Canadian S2 system gives a chance for Russian and some other radio telescopes in the world to be involved into international VLBI programs. Brief descriptions of previous $S 2$ experiments and future possibilities are presented.
\end{abstract}

There are many large radio telescopes in the world not integrated into current VLBI networks because of the lack of modern compatible VLBI recorders. Above all, this situation holds for Russia. Using the Canadian S2 recorder and playback terminals - used mainly for VSOP mission support - gives Russian telescopes a chance to be involved in ground VLBI programs and to achieve a stable position within the VLBI Community. 12 countries have now $S 2$ systems while $S 2$ compatible correlators have been developed in Australia, Canada, Japan and Russia. A few large telescopes (i.e. Bear Lakes RT-64 in Russia, Evpatoria RT-70 in Ukraine, GMRT in India) could join the EVN if $S 2$ enhancement of $M k I V$ correlator at JIVE could be implemented. A first test of $M k I V / S 2$ crossprocessing may be made with the loan of $S 2$ playback and $S 2 / M k I V$ conversion machine from Astro Space Center, Moscow. Secondly, it may be proposed to arrange an ad-hoc $S 2$ VLBI array using the telescopes around the world which are equipped with $S 2$ systems, together with the Penticton $S 2$ correlator. High 

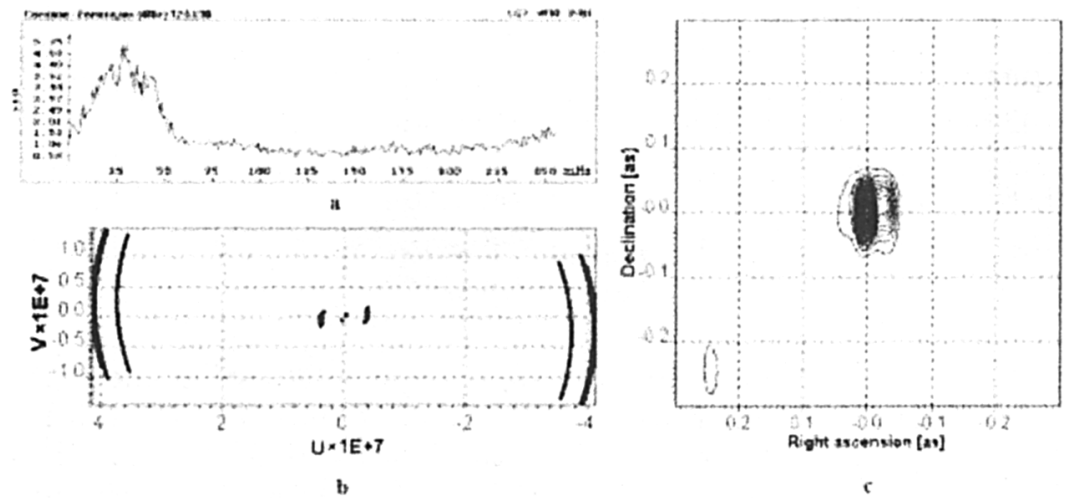

Figure 1. VLBR99.1: fringe for echo signal from Venus for

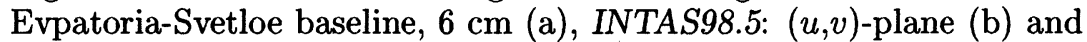
ASL image of $3 \mathrm{C} 309.1,18 \mathrm{~cm} \mathrm{(c)}$

sensitivity (due to Arecibo, GMRT and GBT telescopes) and wide spectrum of baselines up to the largest ones on the Earth could be achieved.

Many successful international VLBI experiments that were carried out since 1993 confirm the feasibility of this $S 2$ initiative. The first international $S 2$ observations were carried out with Parkes RT-64, Hobart RT-26 and Ussuriysk RT-70 in November 1993 (Slysh et al. 1996). The longest S2 baseline 11538 $\mathrm{km}$ was achieved between Bear Lakes and Tidbinbilla in June 1996 (Chuprikov et al. 1996). These data were correlated with Australian ATNF processor at Sydney. Three Russian antennas (Bear Lakes RT-64, Puschino RT-22, Svetloe RT-32) joined with three foreign radio telescopes during observations INTAS98.5 (Arecibo, Green Bank, HartRAO) and INTAS99.4 (HartRAO, Noto, Shanghai) to investigate AGN, solar corona and wind, OH-masers and stars (the data were correlated with Canadian DRAO processor at Penticton). The next such session INTAS00.3 with Noto, Shanghai, GMRT, HartRAO will be carried out on November 28, 2000. The pioneer VLBI radar experiments VLBR99.1 (for Mars and Venus planets) and VLBR00.2 (for Mithra and 2000CE59 asteroids) were organized with use of the planetary radar at Evpatoria RT-70 to develop the new method of investigations of Solar system bodies (test data processing was made by Russian IAA processor at St.-Petersburg).

Some results of INTAS98.5 and VLBR99.1 are presented in Figure 1.

\section{References}

Slysh, V. I., Migenes, V., Kanevsky, B. Z., Molotov, I. E., Samodurov, V. A., Reynolds, J. E., Wilson, W. E., Jauncey, D. L., McCulloch, P. M., Feil, G., and Cannon, W., 1996, MNRAS, 283, L9

Chuprikov, A. A., Likhachev, S. F., Molotov, I. E., et al., 1996, in Proc. of the Technical Workshop for APT and APSG 1996, TWAA96, Kashima, Japan, Dec 10-13, 1996, 289 